\title{
Strategies for efficient and fast wave optics simulation of coded-aperture and other $x$-ray phase-contrast imaging methods
}

\author{
Fabio A. Vittoria, ${ }^{1, *}$ Paul C. Diemoz, ${ }^{1}$ Marco Endrizzi, ${ }^{1}$ Luigi Rigon, ${ }^{2}$ Frances \\ C. Lopez, ${ }^{2,3}$ Diego Dreossi, ${ }^{4}$ Peter R. T. Munro, ${ }^{5,6}$ and Alessandro Olivo ${ }^{1}$ \\ ${ }^{1}$ Department of Medical Physics and Bioengineering, University College London, \\ Malet Place, Gower Street, London WC1E 6BT, UK \\ ${ }^{2}$ INFN, Sezione di Trieste, Via Valerio 2, 34127 Trieste, Italy \\ ${ }^{3}$ Physics Department, University of Trieste, Via Valerio 2, 34127 Trieste, Italy \\ ${ }^{4}$ Sincrotrone Trieste SCpA, S.S. $14 \mathrm{~km}$ 163.5, 34012 Basovizza (TS), Italy \\ ${ }^{5}$ Optical and Biomedical Engineering Laboratory, School of Electrical, \\ Electronic and Computer Engineering, The University of Western Australia, \\ 35 Stirling Highway, Crawley, Western Australia 6009, Australia \\ ${ }^{6}$ Centre for Microscopy, Characterization and Analysis, The University of Western Australia, \\ 35 Stirling Highway, Crawley, Western Australia 6009, Australia
}

compiled: September 30, 2013

\begin{abstract}
We derive a Fourier formulation of coded-aperture x-ray phase contrast imaging, based on the wave theory of optics in the Fresnel approximation. We use this model to develop a very flexible, efficient and general simulation algorithm that can be easily adapted to other implementations of x-ray phase contrast imaging. Likewise, the algorithm enables a simple extension to $2 \mathrm{D}$ aperture designs, different acquisition schemes, etc. Problems related to numerical implementation of the algorithm are analyzed in detail, and simple rules are derived that enable us to avoid or at least mitigate them. Finally, comparisons with experimental data and data obtained with a different simulation algorithm are presented to validate the model and demonstrate its advantages in practical implementations. This also enabled us to demonstrate an increase in computational speed of more than one order of magnitude over previous algorithm.
\end{abstract}

\section{Introduction}

Over recent years, many techniques have been developed to increase the performance of x-ray imaging methods; among these, $\mathrm{x}$-ray phase contrast imaging $(\mathrm{XPCi})$ is one of the most promising and fastest developing. XPCi includes all techniques in which phase effects increase image clarity and contrast $[1,2]$. The list is becoming increasingly longer and includes, among others, Bonse/Hart interferometry $[3,4]$, free-space propagation $[5,6]$, analyser-based methods [7, 8], Talbot $[9,10]$ and Talbot-Lau interferometry [11].

Among all XPCi approaches, the "CodedAperture" (CA) implementation [12] of the edge

\footnotetext{
* Corresponding author: fabio.vittoria.12@ucl.ac.uk
}

illumination [13] XPCi method seems to have features that make it advantageous for practical implementation, related to its ability to work well with standard incoherent x-ray sources [14], reduced exposure times and vibration resilience [15] and easier adaptability to high x-ray energies [16].

The CA XPCi setup is schematically shown in Fig. 1. From the geometrical optics point of view, the operating principle of CA XPCi is easily understood: the first mask (sample mask) creates a series of secondary beams each of which propagates towards a single detector pixel; the second mask (detector mask) intercepts a fixed portion of each beam, so that the intensity measured without a sample would be equal for each pixel (flat field). The fraction of each secondary beam power that reaches the detector is called the illuminated frac- 
tion. When a sample is placed between the two masks, each secondary beam is deflected by an angle $\alpha$ (in the direction $x$ perpendicular to the mask lines) proportional to $\mathrm{d} \phi / \mathrm{d} x$, where $\phi$ is the integral of the difference from unity of the real part of the refractive index of the sample along the x-ray propagation direction; this increases or decreases the signal of each pixel in proportion with $\mathrm{d} \phi / \mathrm{d} x$ [17].

While this description provides an intuitive explanation of the working principle of $\mathrm{CA} \mathrm{XPCi}$, it does not take into account the wave nature of x-rays, the effects of which become stronger as propagation distances, coherence and wavelength increase; a numerical simulation, based on the wave theory of optics, is thus needed.

It should be noticed that the presented formalism for simulations based on wave optics can be easily extended also to other areas of research. To give just one example, in electron diffraction the theoretical description of electron wave function propagating through a sample provided by the multislice method [18] is effectively analogous to the one we discuss in the following section.

In the first part of this paper, we recall the theoretical description of CA XPCi system based on the Fresnel-Kirchoff theory of diffraction and, in the Fresnel approximation, we derive an algorithm capable of simulating a plurality of x-ray imaging systems and, in particular, the CA XPCi method. We then provide a detailed discussion and a series of possible solutions to the implementation problems that are encountered when the imaging method is described through this approach. Finally, we validate our findings through a comparison with both a different CA XPCi simulation algorithm [17], and with experimental data [19]. This shows that a simulation code based on this new model can predict experimental results with high accuracy while achieving a gain of more than one order of magnitude in computation time.

\section{Theory}

A CA XPCi system can be effectively described as a series of free space propagations and transmissions through objects (an "object" being a mask or the sample).

To describe free space propagation, let us consider in the reference frame shown in Fig. 1, a monochromatic wave with wavelength $\lambda$ propagating in the $z$ direction, and let $E_{A}(x, y)$ and $E_{B}(x, y)$ be the complex amplitude of the electric fields on two planes described by $z=z_{A}$ and $z=z_{B}$ respectively, with no objects in between. The Fresnel-

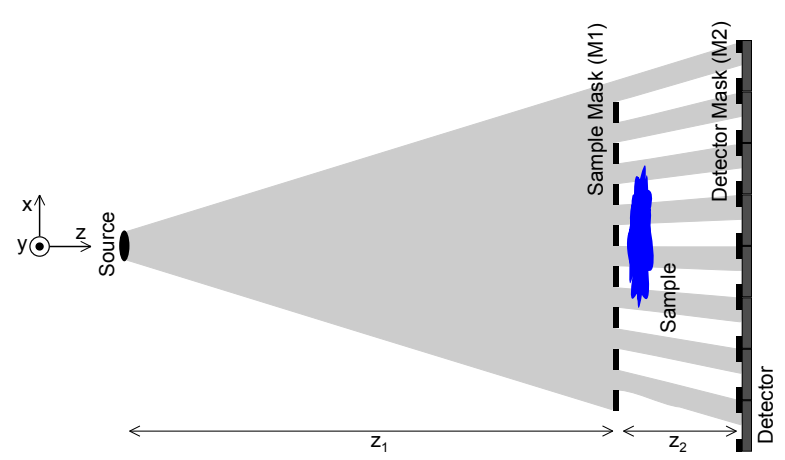

Fig. 1. Schematic diagram of CA XPCi system.

Kirchhoff integral [20] allows us to write:

$$
\begin{aligned}
& E_{B}(x, y)= \\
& =\iint_{-\infty}^{\infty} E_{A}\left(x^{\prime}, y^{\prime}\right) \frac{\exp (-i k|\mathbf{r}|)}{i \lambda|\mathbf{r}|} \cos \alpha \mathrm{d} x^{\prime} \mathrm{d} y^{\prime},
\end{aligned}
$$

with $k=2 \pi / \lambda, \mathbf{r}=\left(x-x^{\prime}\right) \hat{\mathbf{i}}+\left(y-y^{\prime}\right) \hat{\mathbf{j}}+\left(z_{B}-z_{A}\right) \hat{\mathbf{k}}$ and $\alpha$ is the angle between $\mathbf{r}$ and the $z$ axis.

In the Fresnel approximation [20], Eq. (1) becomes

$$
E_{B}(x, y)=E_{A}(x, y) * H_{\Delta z}(x, y),
$$

where $\Delta z=z_{B}-z_{A}, *$ indicates the convolution operation and $H_{z}$ is the Fresnel propagator:

$$
H_{z}(x, y)=\frac{\exp (i k z)}{i \lambda z} \exp \left(i k \frac{x^{2}+y^{2}}{2 z}\right) .
$$

A detailed analysis of the accuracy of the Fresnel approximation can be found in [20] and leads to the conclusion that Eq. (2) is generally valid for distances and wavelengths involved in typical x-ray imaging experiments. For a CA XPCi system, Eq. (2) allows us to describe the free space propagation of $\mathrm{x}$-rays between the source and the sample mask and between the two masks.

In the so called paraxial and thin-object approximations [21], the presence of an object can be taken into account through a complex transfer function $T_{o b j}$ :

$$
E_{\text {out }}(x, y)=T_{\text {obj }}(x, y) E_{\text {in }}(x, y),
$$

where $E_{\text {in }}$ and $E_{\text {out }}$ are the complex amplitudes of the incoming and outgoing fields, respectively. $T_{o b j}$ can be calculated from the complex refractive index of the object, $n=1-\delta+i \beta$, as:

$$
T_{o b j}(x, y)=\exp [-i \phi(x, y)-M(x, y)],
$$


where, if $L$ is the length of the object in the propagation direction $z$,

$$
\begin{aligned}
\phi(x, y) & =k \int_{L} \delta(x, y, z) \mathrm{d} z ; \\
M(x, y) & =k \int_{L} \beta(x, y, z) \mathrm{d} z ;
\end{aligned}
$$

for an ideal mask, $T_{M}(x, y)=1$ within the apertures and $T_{M}(x, y)=0$ in the absorbing septa.

Let us assume, for simplicity's sake, that the system we want to describe has no dependence upon $y$; in this case, neglecting a constant factor arising from the integration over $y$ [17], we can consider the one-dimensional case $(y=0)$. With reference to Fig. 1, it is possible to write the expression for the complex amplitude of the electric field at the detector mask, in the case of a point source placed in the position $x_{s}=0\left(E_{0}(x)=A_{0} \delta_{D}(x)\right.$, where $\delta_{D}$ is the Dirac delta), as:

$$
\begin{aligned}
& E_{M 2}(x)= \\
& =A_{0}\left[H_{1}(x) T_{M 1}(x) T_{o b j}(x)\right] * H_{2}(x),
\end{aligned}
$$

where $H_{1} \equiv H_{z_{1}}, H_{2} \equiv H_{z_{2}}$ and the object and the sample mask are assumed to be in the same plane. In the general case of $x_{s} \neq 0, H_{1}(x)$ must be replaced with $H_{1}\left(x-x_{s}\right)$ to obtain the complex amplitude of the electric field on the detector mask $E_{M 2}\left(x, x_{s}\right)$. Noting that

$$
\begin{aligned}
& H_{z}\left(x-x_{s}\right)= \\
& =\frac{i \lambda z}{\exp (i k z)} H_{z}(x) H_{z}\left(x_{s}\right) \exp \left(-i k x x_{s} / z\right),
\end{aligned}
$$

it is possible to express $E_{M 2}\left(x, x_{s}\right)$ in terms of $E_{M 2}(x)$ as:

$$
E_{M 2}\left(x, x_{s}\right)=C(x) E_{M 2}\left(x+\frac{z_{2}}{z_{1}} x_{s}\right),
$$

where $C(x)$ is a phase factor:

$$
C(x)=\exp \left[i \frac{k}{2 z_{1}}\left(1-\frac{z_{2}}{z_{1}}\right) x_{s}^{2}-i \frac{k}{z_{1}} x x_{s}\right] .
$$

Eq. (9) is important for taking into account an incoherent source of finite size; in this case, in fact, the intensity on the detector can be expressed as:

$$
I_{D}(x)=\int_{-\infty}^{\infty} S\left(x_{s}\right)\left|E_{M 2}\left(x, x_{s}\right) T_{M 2}(x)\right|^{2} \mathrm{~d} x_{s},
$$

where $S\left(x_{s}\right)$ is the source spatial intensity distribution. Indicating by $I_{p}(x)=\left|E_{M 2}(x)\right|^{2}$ the intensity that would be measured on the detector mask with a point source placed in $x_{s}=0$, and with $S_{r}(x)=\left(z_{1} / z_{2}\right) S\left(-x z_{1} / z_{2}\right)$ the rescaled source intensity distribution, Eq. (11) becomes:

$$
I_{D}(x)=\left[S_{r}(x) * I_{p}(x)\right]\left|T_{M 2}(x)\right|^{2}
$$

and the final intensity measured by the n-th pixel will be given by:

$$
I_{n}=\int_{x_{n}}^{x_{n}+P} I_{D}(x) \mathrm{d} x,
$$

where $x_{n}$ is the pixel position and $P$ is the pixel dimension.

By means of simple approximations commonly used in x-ray imaging, it is therefore possible to describe the entire CA XPCi setup by means of a series of convolutions and products. Finally, it is important to remember that the convolution theorem allows calculating convolution products by means of the Fourier Transform (FT):

$$
f(x) * g(x)=\mathcal{F}^{-1}\{\mathcal{F}[f(x)] \mathcal{F}[g(x)]\},
$$

where $\mathcal{F}$ is the FT operator. The convolution theorem plays a basic role in the development of a computationally efficient simulation.

\section{Implementation}

To simulate a CA XPCi experiment it is necessary to solve Eq. (7), Eq. (12) and Eq. (13); this can be done by sampling each of the considered functions and performing convolutions and products numerically. Convolutions can be performed by means of the Fast Fourier Transform (FFT) algorithm, which is computationally very efficient. Nonetheless, it is important to pay attention to some problems that are typically encountered when using this approach.

\section{A. Sampling considerations}

Let us consider a bandlimited function $f(x)$ and let $W_{f}$ be the width of its frequency spectrum. Sampling $f(x)$ at a rate $1 / \Delta x$ implies that its FFT is confined to the frequency range $[-1 /(2 \Delta x), 1 /(2 \Delta x)]$ of width $1 / \Delta x$ : if $1 / \Delta x<W_{f}$ aliasing will occur. One way to avoid aliasing problems is to increase the sampling rate until $1 / \Delta x>$ $W_{f}$. The first step is thus to identify the frequency bandwidth of each function considered in the algorithm in order to set a high enough sampling rate.

Let us consider the Fresnel propagator and its Fourier transform:

$$
\begin{aligned}
& H_{z}(x) \propto \exp \left(i 2 \pi \frac{x^{2}}{2 z \lambda}\right), \\
& \hat{H}_{z}(\xi) \propto \exp \left(-i \pi z \lambda \xi^{2}\right) ;
\end{aligned}
$$


$\hat{H}_{z}(\xi)$ is not bandlimited, which means that it should not be possible to use the FFT without altering the original frequency spectrum. Since $H_{z}(x)$ is an imaginary exponential of argument $x^{2}$, the frequency at which it varies increases with the position $x$; however, the simulated object and masks typically have finite dimensions, so that only a limited portion of space needs to be considered in the simulation. We can therefore assume that the frequency spectrum we are considering is effectively limited.

Mathematically, it is possible to describe the limited portion of space by introducing the $\operatorname{rect}(x)$ function, which is equal to 1 for $|x| \leq 0.5$ and 0 for $|x|>0.5$. If the width of the simulated space is $L$, the function we are dealing with is:

$$
H_{z, e f f}(x) \propto \exp \left(i 2 \pi \frac{x^{2}}{2 z \lambda}\right) \operatorname{rect}\left(\frac{x}{L}\right)
$$

and thus

$$
\begin{aligned}
& \hat{H}_{z, e f f}(\xi) \propto \exp \left(-i \pi z \lambda \xi^{2}\right) * \frac{\sin (\pi L \xi)}{\pi \xi}= \\
& =\int_{-\infty}^{\infty} \exp \left[-i \pi z \lambda(\xi-\eta)^{2}\right] \frac{\sin (\pi L \eta)}{\pi \eta} \mathrm{d} \eta .
\end{aligned}
$$

We are interested in calculating $\hat{H}_{z, e f f}(\xi)$ for high frequency values (i.e. $\gg 1 / L)$ and, at the same time, due to the $\sin (\pi L \eta) /(\pi \eta)$ term, the main contribution inside the integral is due to values of $\eta$ of the order of $1 / L$. We can then make the approximation $(\xi-\eta)^{2} \approx \xi^{2}-2 \xi \eta$ for small values of $\eta$, and the modulus of Eq. (17) becomes

$$
\begin{aligned}
\left|\hat{H}_{z, e f f}(\xi)\right| & \propto\left|\int_{-\infty}^{\infty} \exp (i 2 \pi z \lambda \xi \eta) \frac{\sin (\pi L \eta)}{\pi \eta} \mathrm{d} \eta\right|= \\
& =\operatorname{rect}\left(\frac{z \lambda \xi}{L}\right) .
\end{aligned}
$$

The convolution product in the Fourier space acts here as a "low-pass" filter with a cut-off frequency $\xi_{\text {cut }}=L /(2 z \lambda)$.

Fig. 2 shows a comparison between the functions $\left|\hat{H}_{z, e f f}(\xi)\right|$ (calculated numerically) and $\operatorname{rect}(z \lambda \xi / L)$; the approximation made to perform the analytical calculation does not allow describing the oscillations of $\left|\hat{H}_{z, \text { eff }}(\xi)\right|$ around its mean value, but it does allow the correct derivation of its average behaviour. Furthermore, it provides an important parameter, $\xi_{c u t}=L /(2 z \lambda)$, which is the frequency at which $\left|\hat{H}_{z, \text { eff }}(\xi)\right|$ reaches half its average value near $\xi=0$. For frequency values greater than $2 \xi_{\text {cut }},\left|\hat{H}_{z, \text { eff }}(\xi)\right|$ can be effectively considered equal to 0 ; for this reason we consider the frequency bandwidth of the effective Fresnel propagator to be:

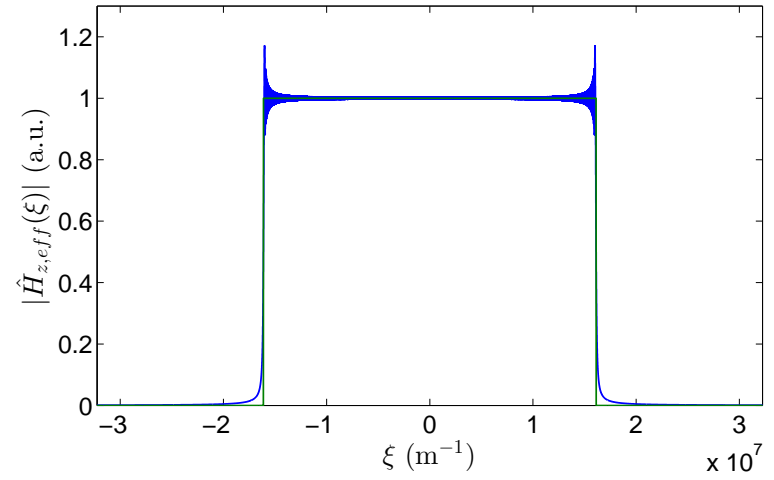

Fig. 2. Modulus of $\hat{H}_{z, \text { eff }}(\xi)$ : the blue curve is calculated numerically, while the green curve is calculated analytically with the approximation discussed in the text. Parameters used in the simulation: $z=2 \mathrm{~m}, L=2 \mathrm{~mm}$, $\lambda=0.31 \AA(E=40 \mathrm{KeV})$.

$$
W_{H}=2 L /(z \lambda) .
$$

Let us consider now the complex transfer function of a sample mask with a single aperture of width $A$ and its Fourier transform:

$$
\begin{aligned}
& T_{M}(x)=\operatorname{rect}\left(\frac{x}{A}\right), \\
& \hat{T}_{M}(\xi)=\frac{\sin (\pi A \xi)}{\pi \xi} ;
\end{aligned}
$$

$\hat{T}_{M}(\xi)$ is also a non-bandlimited function. However, $\left|\hat{T}_{M}(\xi)\right|<10^{-3}\left|\hat{T}_{M}(0)\right|$ for $\xi>10^{3} /(\pi A)$; the error made by neglecting frequencies greater than $10^{3} /(\pi A)$ can be therefore considered negligible. This leads us to set:

$$
W_{M}=\frac{2 \times 10^{3}}{\pi A} ;
$$

note that the above condition also holds for the general case of a mask with several apertures of width $A$.

Let us now consider a general object obtained as the convolution of a rect function with a Gaussian function and its Fourier transform:

$$
\begin{aligned}
& T_{o b j}(x) \propto \exp \left(-\frac{x^{2}}{2 \sigma_{o}^{2}}\right) * \operatorname{rect}\left(\frac{x}{O}\right), \\
& \hat{T}_{o b j}(\xi) \propto \exp \left(-2 \pi^{2} \sigma_{o}^{2} \xi^{2}\right) \frac{\sin (\pi O \xi)}{\pi \xi} ;
\end{aligned}
$$

the rect function allows us to take into account the object dimension $O$, while the Gaussian function does the same for its smoothness through $\sigma_{o}$. Following the previous argument, 
$\left|\hat{T}_{o b j}(\xi)\right|<10^{-3}\left|\hat{T}_{o b j}(0)\right|$ if $\xi>10^{3} /(\pi O)$ and/or $\xi>\left[3 \ln 10 /\left(2 \pi \sigma_{o}^{2}\right)\right]^{1 / 2}$. We can then set:

$$
W_{o b j}=\min \left\{\frac{2 \times 10^{3}}{\pi O},\left[\frac{6 \ln 10}{\pi \sigma_{o}^{2}}\right]^{1 / 2}\right\} .
$$

Comparing Eq. (21) and Eq. (23), we can see that $W_{M}$ is greater than $W_{o b j}$ if $A<O$ and/or if $A \lesssim 300 \sigma_{o}$. In a typical CA XPCi system, $A \approx 1 \div 50 \mu \mathrm{m}$ and we can therefore conclude that, for objects with micrometric internal structures, $W_{o b j}<W_{M}$; hereinafter this condition will be assumed to be true.

Let us finally consider a Gaussian distributed source with standard deviation $\sigma_{s}$. The rescaled source intensity distribution is then:

$$
\begin{aligned}
& S_{r}(x) \propto \exp \left(-\frac{x^{2}}{2 \sigma_{r}^{2}}\right), \\
& \hat{S}_{r}(\xi) \propto \exp \left(-2 \pi^{2} \sigma_{r}^{2} \xi^{2}\right) ;
\end{aligned}
$$

where $\sigma_{r}=\left(z_{2} / z_{1}\right) \sigma_{s}$. Following the previous argument, it is then possible to set:

$$
W_{S r}=\left[\frac{6 \ln 10}{\pi \sigma_{r}^{2}}\right]^{1 / 2} .
$$

Studying every function separately does not ensure that aliasing problems are avoided; when we multiply two functions, in fact, the frequency bandwidth changes. Let us consider two bandlimited function $f(x)$ and $g(x)$ and the product $h(x)=$ $f(x) g(x)$; in the Fourier space $\hat{h}(\xi)=\hat{f}(\xi) * \hat{g}(\xi)$ and $W_{h}=W_{f}+W_{g}$. In this case it is then necessary that $\left(W_{f}+W_{g}\right)<1 / \Delta x$. Taking into account all the multiplications in the algorithm, one obtains the following conditions:

$$
\begin{gathered}
\max \left(W_{H 1}+2 W_{M 1}, W_{H 2}, W_{S r}\right)<1 / \Delta x, \\
W_{I p}=2 \min \left(W_{H 1}+2 W_{M 1}, W_{H 2}\right)<1 / \Delta x, \\
\min \left(W_{S r}, W_{I p}\right)+2 W_{M 2}<1 / \Delta x .
\end{gathered}
$$

In the first inequality the term $W_{H 1}+2 W_{M 1}$ is derived from the multiplication of $H_{1}, T_{M 1}$ and $T_{o b j}$ in Eq. (7), having applied the condition $W_{o b j}<W_{M 1}$; $W_{H 2}$ and $W_{S r}$ take into account $H_{2}$ and $S_{r}$ in Eq. (7) and Eq. (12), respectively. In the second equation, $W_{I p}$ is the bandwidth of $I_{p}$ : the factor of 2 derives from the square modulus of $E_{M 2}$, while the minimum between $W_{H 1}+2 W_{M 1}$ and $W_{H 2}$ is taken because of the convolution product in Eq. (7). The last equation derives from Eq. (12).

Fig. 3 shows a comparison among various simulations of the same experimental conditions, the only

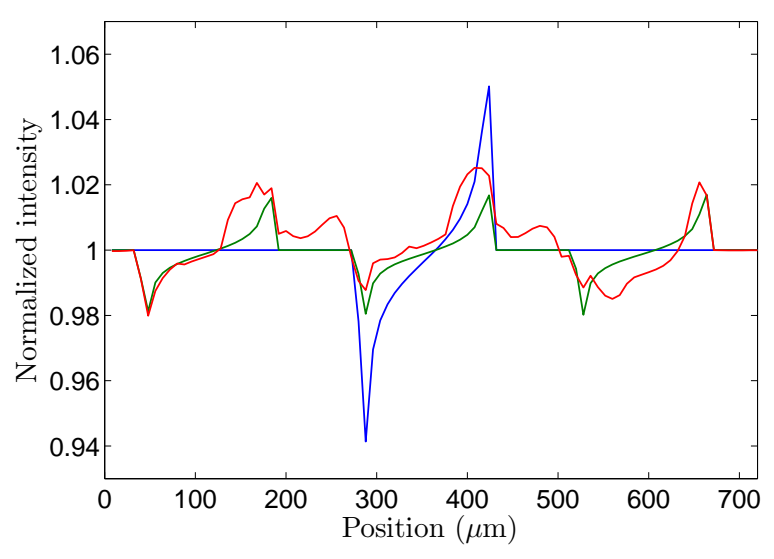

Fig. 3. Simulations of CA XPCi profiles of a polypropylene wire, obtained with different numbers of sampling points: $\delta x_{\text {blue }}=3.4 \mathrm{~nm}$ is derived in accordance with Eq. $(26), \delta x_{\text {green }}=15 \delta x_{\text {blue }}, \delta x_{\text {red }}=30 \delta x_{\text {blue }}$. Only the first simulation (blue) provides correct results. Parameters used in the simulation: monochromatic Gaussian distributed source $(F W H M=60 \mu \mathrm{m}, E=30 \mathrm{KeV}) ; z_{1}$ $=1.6 \mathrm{~m}, z_{2}=0.4 \mathrm{~m}$; sample mask with $12 \mu \mathrm{m}$ apertures and a period of $80 \mu \mathrm{m}$; detector mask with $20 \mu \mathrm{m}$ apertures and a period of $100 \mu \mathrm{m} ; 50 \%$ illuminated fraction; pixel size $=100 \mu \mathrm{m}$; wire diameter $=140 \mu \mathrm{m}$; number of dithering steps (number of sub-pixel sample displacements $)=10($ each step $=8 \mu \mathrm{m})$.

difference being the sampling rate. As the sampling rate decreases, aliasing increasingly alters the original signal and only when the conditions expressed in Eq. (26) are satisfied, correct results are obtained from the simulation.

\section{B. Circular convolution considerations}

Let us consider Eq. (14) and let $f_{l}=f(l \Delta x)$ and $g_{l}=g(l \Delta x)$ be sampled version of the functions $f$ and $g$, with $-N / 2 \leq l \leq N / 2-1$; where the number of sampling points $N$ is assumed to be even. The discretized version of the convolution theorem can be expressed as:

$$
f_{l} \bar{*} g_{l}=F F T^{-1}\left\{F F T\left[f_{l}\right] F F T\left[g_{l}\right]\right\} ;
$$

where $\bar{*}$ indicates a type of discrete convolution operation, called circular convolution. Circular convolution implicitly assumes periodicity at the boundaries of the sampled space: rather than a sampled version of $f * g, f_{l} \bar{*} g_{l}$ is a sampled version of $f_{p} * g_{p}$, where $f_{p}$ is a periodic function consisting of the repetition of a series of infinite copies of $f(x) \operatorname{rect}(x / L)$ shifted by the length $L=N \Delta x$ of the sampled space, i.e. $f_{p}(x)=\sum_{n} f(x-n L) \operatorname{rect}(x / L-n)$, while $g_{p}(x)=g(x) \operatorname{rect}(x / L)$. The presence of the $\operatorname{rect}(x / L)$ term in the previous equations derives 
from having sampled a limited portion of space of width L.

Thus, the result of the discrete convolution between $f$ and $g$, performed through the discrete Fourier transform, is $f_{p} * g_{p}$ rather that $f * g$. To obtain correct results from the simulation, it is thus necessary to understand in which circumstances $f_{p} * g_{p}=f * g$.

Let the support of $f$ and $g$ be finite and let $R_{f}$ and $R_{g}$ be the widths of their support; if the support of one of the functions, for example $f$, is not finite or if it is greater than $L$, the sampling procedure allows us to consider $R_{f}=L$. When $R_{f}+R_{g}<L$, $f_{p} * g_{p}=f * g$ in every point of the sampled space. If the last condition is not met, $f_{p} * g_{p} \neq f * g$ in two regions at the boundaries of the sampled space, where the different copies in $f_{p}$ interfere with each other due to the convolution with $g_{p}$; the total width of these regions is equal to $R_{f}+R_{g}-L$.

In the case of our simulations, two convolution products are performed in Eq. 7 and in Eq. 12. In Eq. 7 the support of $H_{1} T_{M 1} T_{o b j}$ is equal to the dimension of the sample mask $R_{M 1}$, while $H_{2}$ extends over the entire sampled space $L$; in this condition, the width of the region of error is equal to $R_{M 1}$. In Eq. 12 the support $R_{S r}$ of the rescaled source intensity distribution $S_{r}$ is limited due to the finite source size, while $I_{p}$ extends over the entire sampled space $L$; the width of the region of error here is then equal to $R_{S r}$. Let $L_{D}$ be the width of the detector we want to simulate. In order for the simulation to give a correct result, it is then necessary to extend the sampled space width $L$ until the entire detector length $L_{D}$ is contained in the zone within which circular and conventional convolution provide the same result, so that Eq. (13) can be evaluated correctly. It is easy to demonstrate that, if $L>L_{D}+R_{M 1}+R_{S r}$, the simulation gives correct results.

Fig. 4 shows a comparison between results obtained by simulating the same experimental conditions, with the only difference being the sampled space. Artefacts induced by circular convolution are evident in the profile calculated using an insufficient sampled space (green curve), while they do not affect the profile calculated with a sufficiently large sampled space (blue curve). By appropriately handling sampling and circular convolution problems along the lines described above, we can ensure that numerical implementation errors do not affect the results of our simulations.

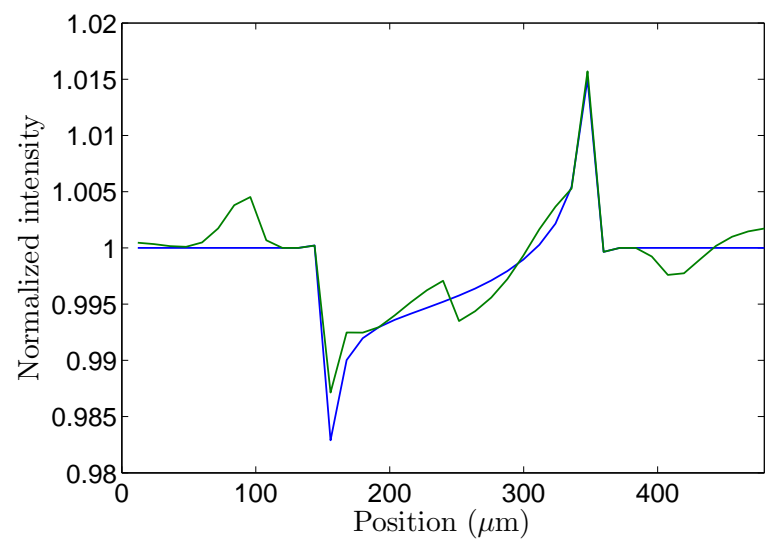

Fig. 4. Simulations of CA XPCi profiles of a polypropylene wire, obtained using different dimensions for the sampled space. The blue curve is calculated considering a sampled space $L=L_{D}+R_{M 1}+R_{S r}$, which leads to correct results; the green curve is calculated with a sampled space $L=L_{D}$, which causes errors in the simulated profile. Parameters used in the simulation: monochromatic Gaussian distributed source $(F W H M=1 \mu \mathrm{m}, E$ $=30 \mathrm{KeV}) ; z_{1}=100 \mathrm{~m}, z_{2}=0.1 \mathrm{~m}$; sample mask with $20 \mu \mathrm{m}$ apertures and a period of $120 \mu \mathrm{m}$; detector mask with $20 \mu \mathrm{m}$ apertures and a period of $120 \mu \mathrm{m} ; 50 \%$ illuminated fraction; pixel size $=120 \mu \mathrm{m}$; wire diameter $=160 \mu \mathrm{m}$; number of dithering steps $=10$ (each step $=$ $12 \mu \mathrm{m})$.

\section{Comparison with another algorithm}

In this section, we compare our algorithm with the one described in reference [17]. Both algorithms are based on wave optics, but strongly differ for the way in which the model is implemented.

Although different samples and acquisition conditions are considered in Figs. 5(a,b), no appreciable differences can be seen between the results provided by the two algorithms. Such comparison has been repeated for a large number of cases simulating a wide range of different experimental conditions, and the same agreement was obtained. This is an expected result, as the algorithm described in reference [17] was validated experimentally several times; rather than different results, we are aiming here for achieving the same results with a substantial gain in computation time, as well as higher flexibility and adaptability to different experimental conditions.

Fig. 6 shows the ratio between the computation times required by the two algorithms as a function of the dimensions of the simulated sample: for the investigated cases, the time reduction obtained through the new algorithm was between 20 and 110 fold. The algorithm in [17], in fact, uses Gaussian 


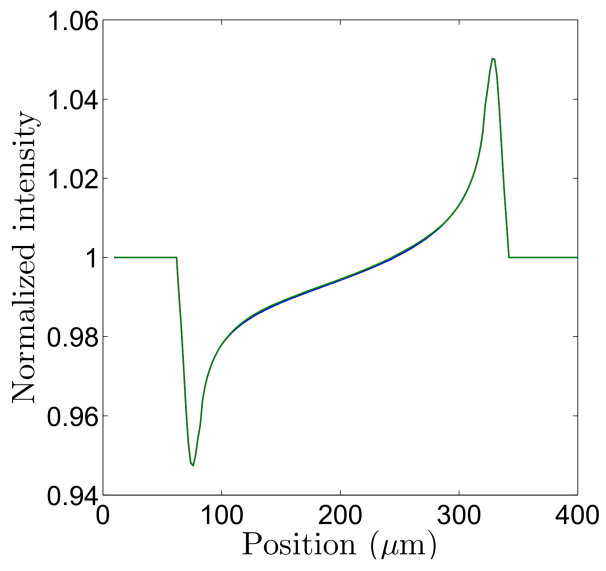

(a)

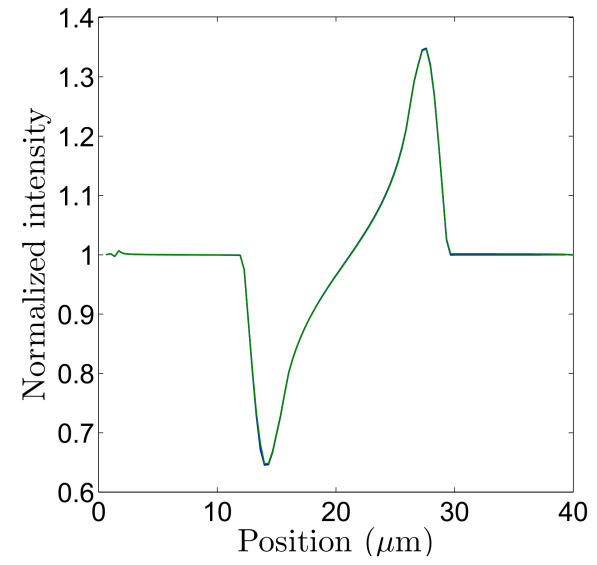

(b)

Fig. 5. Comparison between simulated results obtained with the proposed algorithm (blue curves) and the one described in [17] (green curves). (a) Polypropylene wire: monochromatic Gaussian distributed source ( $F W H M=$ $60 \mu \mathrm{m}, E=30 \mathrm{KeV}) ; z_{1}=1.6 \mathrm{~m}, z_{2}=0.4 \mathrm{~m}$; sample mask with $20 \mu \mathrm{m}$ apertures and $80 \mu \mathrm{m}$ period; detector mask with $50 \mu \mathrm{m}$ apertures and $100 \mu \mathrm{m}$ period; $50 \%$ illuminated fraction; pixel size $=100 \mu \mathrm{m}$; wire diameter $=260$ $\mu \mathrm{m}$; number of dithering steps $=40$ (each step $=2 \mu \mathrm{m}$ ). (b) Aluminium wire: monochromatic Gaussian distributed source $(F W H M=1 \mu \mathrm{m}, E=20 \mathrm{KeV}) ; z_{1}=0.1 \mathrm{~m}, z_{2}=1 \mathrm{~m}$; sample mask with $3.4 \mu \mathrm{m}$ apertures and $13.6 \mu \mathrm{m}$ period; detector mask with $75 \mu \mathrm{m}$ apertures and $150 \mu \mathrm{m}$ period; $50 \%$ illuminated fraction; pixel size $=150 \mu \mathrm{m}$; wire diameter $=14 \mu \mathrm{m}$; dithering steps $=40($ each step $=0.34 \mu \mathrm{m})$.

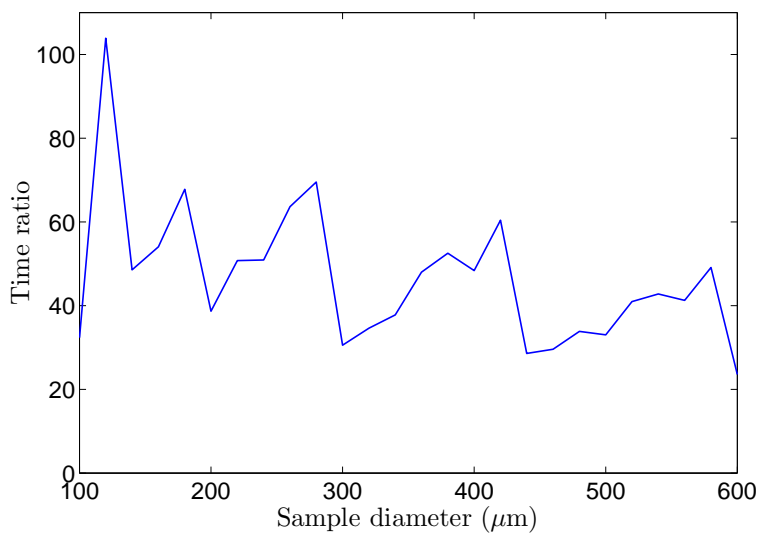

Fig. 6. Ratio between the computational times for the algorithm described in [17] and the one presented here, as a function of the sample dimension. Parameters used in the simulation: monochromatic Gaussian distributed source $(F W H M=60 \mu \mathrm{m}, E=30 \mathrm{KeV}) ; z_{1}=1.6 \mathrm{~m}, z_{2}$ $=0.4 \mathrm{~m}$; sample mask with $40 \mu \mathrm{m}$ apertures and $80 \mu \mathrm{m}$ period; detector mask with $50 \mu \mathrm{m}$ apertures and $100 \mu \mathrm{m}$ period; $50 \%$ illuminated fraction; pixel size $=100 \mu \mathrm{m}$; polypropylene wire sample; dithering steps $=5$ (each step $=16 \mu \mathrm{m})$.

quadrature numerical integration to solve diffraction integrals, which is computationally inefficient compared to computing convolution products using FFT.

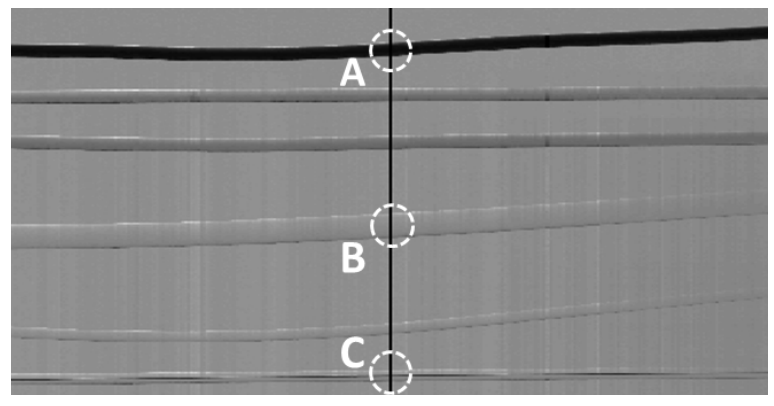

Fig. 7. Image of different wires acquired with synchrotron radiation using the $\mathrm{CA}$ XPCi method.

The gain in terms of computational time is higher than one order of magnitude, which is crucial when the sample dimension increases or when it is necessary to simulate a large number of images like, for example, in computed tomography.

\section{Comparison with experimental data}

Finally, we compare the results of our simulation with experimental measurements performed at the SYRMEP beamline of the Elettra synchrotron facility (Trieste, Italy). A detailed description of the used experimental setup and acquisition conditions can be found in reference [19].

Fig. 7 shows an image acquired for a series of different wires. These were chosen as their symme- 

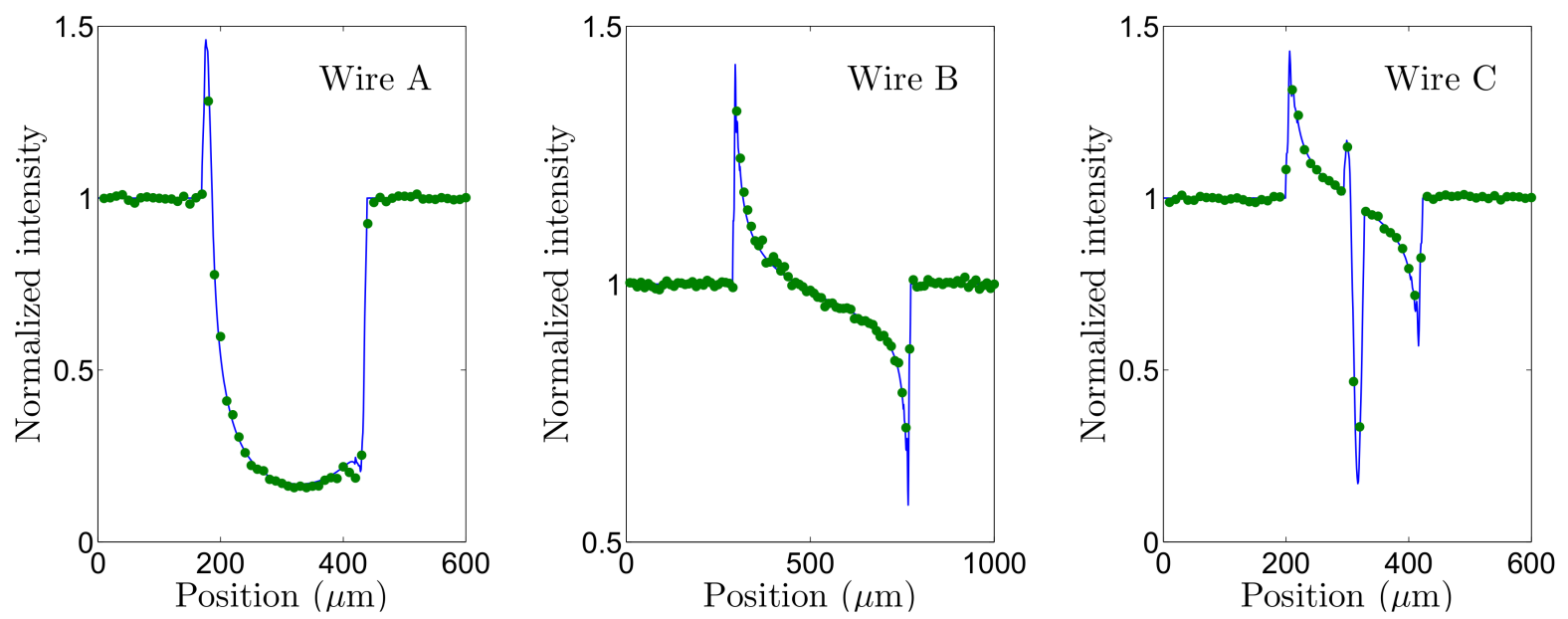

Fig. 8. Comparison between experimental data (intensity profiles along the black vertical line in Fig. 7) and simulation results.

Table 1. Properties of wires highlighted in Fig. 7.

\begin{tabular}{cccccc}
\hline Wire & Material & $\begin{array}{c}\text { Nominal } \\
\text { diameter }(\mu \mathrm{m})\end{array}$ & $\begin{array}{c}\text { Estimated } \\
\text { diameter }(\mu \mathrm{m})\end{array}$ & $\delta[22]$ & $\beta[22]$ \\
\hline $\mathrm{A}$ & Titanium $^{2}$ & $250 \pm 10 \%$ & 250 & $2.19 \times 10^{-6}$ & $3.46 \times 10^{-8}$ \\
$\mathrm{~B}$ & PEEK $^{\mathrm{a}}$ & $450 \pm 20 \%$ & 464 & $7.15 \times 10^{-7}$ & $2.74 \times 10^{-10}$ \\
$\mathrm{C}$ & Boron $^{\mathrm{b}}$ & $200 \pm 20 \%$ & 204 & $1.12 \times 10^{-6}$ & $2.84 \times 10^{-10}$ \\
\hline
\end{tabular}

${ }^{a}$ PEEK stands for polyetheretherketone.

b The boron wire contains a thin tungsten core with an estimated diameter of $14 \mu \mathrm{m}$.

try properties enable a straightforward comparison with the one-dimensional simulation approach described here.

The vertical FWHM of the virtual x-ray source was $80 \mu \mathrm{m}$, and a $\mathrm{Si}(1,1,1)$ crystal reflection was used to select a quasi-monochromatic beam with an energy of $20 \mathrm{KeV}$ (bandwidth 0.2\%). The image was acquired through a scanning procedure [19], and two single slits were used as sample and detector masks with apertures in the vertical direction equal to $20 \mu \mathrm{m}$ and $150 \mu \mathrm{m}$, respectively. The source to sample distance was $20 \mathrm{~m}$, the sample to detector distance was $0.55 \mathrm{~m}$, and the detector featured a single row of pixels with dimensions equal to $300 \times 50 \mu \mathrm{m}^{2}$ in the vertical and horizontal directions, respectively. The specifications for the wires highlighted with white circles in Fig. 7 are listed in Table 1.

To test the developed method under a wide range of conditions, we selected wires with very different characteristics: titanium presents very strong absorption, PEEK is almost transparent, and the boron wire presents an additional complication in the fact that it contains a tungsten core. The comparison between the intensity measured along the black vertical line in Fig. 7 and the intensity simulated with our code is shown in Fig. 8; a very good agreement is found for all the considered wires.

\section{Conclusion}

We have presented a simulation algorithm, based on Fresnel wave optics, capable of simulating CA $\mathrm{XPCi}$ systems. This method is very general and can therefore be easily adapted to a wide range of other x-ray imaging techniques. Numerical implementation problems such as sampling rate and sampled space have been studied in detail, and simple rules to avoid simulation errors and artefacts have been provided.

Comparisons with both a previously validated, different algorithm [17] and experimental data [19] have been performed, resulting in very good agreement in both cases. The presented algorithm is therefore able to accurately predict experimental results, and presents the advantage of a gain greater than one order of magnitude in terms of computa- 
tion time compared to previous implementations.

Finally, we would like to point out that Eq. (7), Eq. (12), Eq. (13) and Eq. (14) can be used to describe a two-dimensional system by just adding the dependency on the $y$ coordinate, performing the integration in Eq. (13) over the entire pixel area and considering the two-dimensional FT in Eq. (14). In this case, each function is sampled along $x$ and $y$ at sampling rates $1 / \Delta x$ and $1 / \Delta y$ respectively, and an area $L_{x} \times L_{y}$ is considered in the simulation. The conditions introduced in section 3 for the sampling rate $1 / \Delta x$ and the length $L_{x}$ still hold, provided that each term $W_{f}$ is defined as the maximum width of the frequency spectrum of $f$ along the first coordinate in the Fourier space, and each term $R_{f}$ is equal to the maximum width of the support of $f$ along the coordinate $x$. Following the same procedure, it is straightforward to derive equivalent conditions for $1 / \Delta y$ and $L_{y}$. The main practical limitation in this case would be the additional computational requirements (memory, etc.), which could be very demanding; however, we would still expect the proposed method to be more computationally efficient than the other approach mentioned in the paper.

\section{Acknowledgments}

This work is funded by the EPSRC (Grants $\mathrm{EP} / \mathrm{G} 004250 / 1$ and EP/I021884/1). F.C.L. is supported by the Prof. Giulio Brautti PhD Memorial Fellowship. P.R.T.M. is supported by a Discovery Early Career Research Award from the Australian Research Council (DE120101331).

\section{References}

[1] A. Olivo, "Recent patents in x-ray phase contrast imaging," Rec. Pat. Biomed. Eng. 3, 95-106 (2010).

[2] A. Bravin, P. Coan and P. Suortti, "X-ray phasecontrast imaging: from pre-clinical applications towards clinics," Phys. Med. Biol. 58, R1-R35 (2013).

[3] U. Bonse and M. Hart, "An x-ray interferometer," Appl. Phy. Lett. 6, 155-6 (1965).

[4] A. Momose, T. Takeda, Y. Itai and K. Hirano, "Phase-contrast x-ray computed tomography for observing biological soft tissues," Nat. Med. 2, 4735 (1996).

[5] A. Snigirev, I. Snigireva, V. Kohn, S. Kuznetsov and I. Schelokov, "On the possibilities of x-ray phase contrast microimaging by coherent high-energy synchrotron radiation," Rev. Sci. Instrum. 66, 5486-92 (1995).

[6] S. W. Wilkins, T. E. Gureyev, D. Gao, A. Pogany and A. W. Stevenson, "Phase-contrast imaging using polychromatic hard x-rays," Nature $\mathbf{3 8 4}, 335-8$ (1996).
[7] V. N. Ingal and E. A. Beliaevskaya, "X-ray planewave topography observation of the phase contrast from a non-crystalline object," J. Phys. D: Appl. Phys. 28, 2314-17 (1995).

[8] D. Chapman, W. Thomlinson, R. E. Johnston, D. Washburn, E. Pisano, N. Gmur, Z. Zhong, R. Menk, F. Arfelli and D. Sayers, "Diffraction enhanced xray imaging," Phys. Med. Biol. 42, 2015-25 (1997).

[9] C. David, B. Nohammer, H. H. Solak and E. Ziegler, "Differential x-ray phase contrast imaging using a shearing interferometer," Appl. Phys. Lett. 81, 3287-9 (2002).

[10] A. Momose, S. Kawamoto, I. Koyama, Y. Hamaishi, K. Takai and Y. Suzuki, "Demonstration of x-ray Talbot interferometry," Jpn. J. Appl. Phys. 42, L866-8 (2003).

[11] F. Pfeiffer, T. Weitkamp, O. Bunk and C. David, "Phase retrieval and differential phase-contrast imaging with low-brilliance x-ray sources," Nat. Phys. 2, 258-61 (2006).

[12] A. Olivo and R. Speller, "A coded-aperture approach allowing $\mathrm{x}$-ray phase contrast imaging with conventional sources," Appl. Phys. Lett. 91, 074106 (2007).

[13] A. Olivo, F. Arfelli, G. Cantatore, R. Longo, R. H. Menk, S. Pani, M. Prest, P. Poropat, L. Rigon, G. Tromba, E. Vallazza and E. Castelli, "An innovative digital imaging set-up allowing a low-dose approach to phase contrast applications in the medical field," Med. Phys. 28, 1610-9 (2001).

[14] P. R. T. Munro, K. Ignatyev, R. D. Speller and A. Olivo, "Phase and absorption retrieval using incoherent X-ray sources," Proc. Natl. Am. Sci. 109, 13922-27, (2012).

[15] A. Olivo, K. Ignatyev, P. R. T. Munro and R. D. Speller, "Noninterferometric phase-contrast images obtained with incoherent x-ray sources," Appl. Opt. 50, 1765-9 (2011).

[16] K. Ignatyev, P. R. T. Munro, D. Chana, R. D. Speller and A. Olivo, "Coded apertures allow highenergy x-ray phase contrast imaging with laboratory sources," J. Appl. Phys. 110, 014906 (2011).

[17] P. R. T. Munro, K. Ignatyev, R. D. Speller and A. Olivo, "The relationship between wave and geometrical optics model of coded aperture type x-ray phase contrast imaging systems," Opt. Expr. 18, 4103-17 (2010).

[18] J.M. Cowley and A.F. Moodie, "The scattering of electrons by atoms and crystals. I. A new theoretical approach," Acta Cryst. 10, 609-19 (1957).

[19] P. R. T. Munro, L. Rigon, K. Ignatyev, F. C. Lopez, D. Dreossi, R. D. Speller and A. Olivo, "A quantitative, non-interferometric X-ray phase contrast imaging technique," Opt. Expr. 21, 647-61 (2013).

[20] J. W. Goodman, Introduction to Fourier Optics (McGraw-Hill, 1996). 
[21] D. M. Paganin, Coherent X-Ray Optics (Oxford University Press, 2006).

[22] B. Henke, E. Gullikson and J. Davis, "X-Ray Inter- actions: Photoabsorption, Scattering, Transmission and Reflection at $\mathrm{E}=50-30,000 \mathrm{eV}, \mathrm{Z}=1-92$," At. Data Nucl. Data Tables 54, 181-342 (1993). 